\title{
A Universal Inequality for Axisymmetric and Stationary Black Holes with Surrounding Matter in the Einstein-Maxwell Theory
}

\author{
Jörg Hennig ${ }^{1}$, Carla Cederbaum ${ }^{1}$, Marcus Ansorg ${ }^{1,2}$ \\ ${ }^{1}$ Max Planck Institute for Gravitational Physics, Am Mühlenberg 1, D-14476 Golm, Germany. \\ E-mail: Joerg.Hennig@ aei.mpg.de, Carla.Cederbaum@aei.mpg.de \\ 2 Institute of Biomathematics and Biometry, Helmholtz Zentrum München, Ingolstädter Landstr. 1, D-85764 \\ Neuherberg, Germany. E-mail: marcus.ansorg @ helmholtz-muenchen.de
}

Received: 15 December 2008 / Accepted: 29 April 2009

Published online: 1 September 2009 - ( $)$ The Author(s) 2009. This article is published with open access at Springerlink.com

Abstract: We prove that in Einstein-Maxwell theory the inequality $(8 \pi J)^{2}+\left(4 \pi Q^{2}\right)^{2}<$ $A^{2}$ holds for any sub-extremal axisymmetric and stationary black hole with arbitrary surrounding matter. Here $J, Q$, and $A$ are angular momentum, electric charge, and horizon area of the black hole, respectively.

\section{Introduction}

For a single rotating, electrically charged, axisymmetric and stationary black hole in vacuum (described by the Kerr-Newman family of solutions), the angular momentum $J$, the electric charge $Q$, and the horizon area $A$ are restricted by the inequality

$$
p_{J}^{2}+p_{Q}^{2} \leq 1 \quad \text { with } \quad p_{J}:=\frac{8 \pi J}{A}, \quad p_{Q}:=\frac{4 \pi Q^{2}}{A} .
$$

Equality in (1) holds if and only if the Kerr-Newman black hole is extremal. That is to say,

$$
p_{J}^{2}+p_{Q}^{2}<1
$$

holds for any non-extremal Kerr-Newman black hole.

As was shown in [1], the equality $p_{J}^{2}+p_{Q}^{2}=1$ holds more generally in EinsteinMaxwell theory for axisymmetric and stationary degenerate ${ }^{1}$ black holes with surrounding matter. Moreover, it was conjectured in [1] that inequality (1) is still valid if the black hole is surrounded by matter (i.e. if it is not a member of the Kerr-Newman family).

Inequality (2) was proved in [7] for axisymmetric and stationary black holes with surrounding matter in pure Einsteinian gravity (without Maxwell field). In that article, emphasis was put on "physically relevant" configurations by assuming the black hole to

\footnotetext{
1 Degeneracy of an axisymmetric and stationary black hole is defined by vanishing surface gravity $\kappa$.
} 
be sub-extremal. This condition requires the existence of trapped surfaces (i.e. surfaces with a negative expansion of outgoing null geodesics) in every sufficiently small interior vicinity of the event horizon, see [3]. Here, we consider again sub-extremal axisymmetric and stationary black holes with arbitrary surrounding matter, but provide a proof of (2) which is valid in the full Einstein-Maxwell theory.

The idea of the proof relies on showing that a black hole cannot be sub-extremal for $p_{J}^{2}+p_{Q}^{2} \geq 1$. In order to prove this, we study the Einstein-Maxwell equations in a vicinity of the black hole horizon. It turns out that a reformulation can be found which states that an appropriate functional $I$ (to be defined below) must always be greater than or equal to 1 . In this way, we encounter a variational problem, and the corresponding solution provides a proof of inequality (2). As will be shown below, this variational problem can be treated with methods from the calculus of variations.

This paper is organized as follows. In Sec. 2, we introduce appropriate coordinates which are adapted to the subsequent analysis. Moreover, we list the Einstein-Maxwell equations and the corresponding boundary and regularity conditions in these coordinates. In Sec. 3, we express the ingredients $p_{J}$ and $p_{Q}$, which appear in the inequality (2), in terms of metric and electromagnetic potentials. We formulate the variational problem mentioned above in Sec. 4 and solve it in Sec. 5. Finally, we conclude this paper with a discussion on physical implications of inequality (2), see Sec. 6. In an appendix, we establish a connection to degenerate black holes.

\section{Coordinate Systems and Einstein Equations}

Following Bardeen [2], we describe an exterior electrovacuum vicinity of the black hole ${ }^{2}$ in spherical coordinates $(R, \theta, \varphi, t)$ in terms of a Boyer-Lindquist-type ${ }^{3}$ line element

$$
\mathrm{d} s^{2}=\hat{\mu}\left(\frac{\mathrm{d} R^{2}}{R^{2}-r_{\mathrm{h}}^{2}}+\mathrm{d} \theta^{2}\right)+\hat{u} \sin ^{2} \theta(\mathrm{d} \varphi-\omega \mathrm{d} t)^{2}-\frac{4}{\hat{u}}\left(R^{2}-r_{\mathrm{h}}^{2}\right) \mathrm{d} t^{2},
$$

where the metric potentials $\hat{\mu}, \hat{u}$ and $\omega$ are functions of $R$ and $\theta$ alone and where in addition $\hat{\mu}$ and $\hat{u}$ are positive functions. The event horizon $\mathcal{H}$ is located at $R=r_{\mathrm{h}}$, $r_{\mathrm{h}}=$ constant $>0$.

The electromagnetic field gives rise to an energy momentum tensor

$$
T_{i j}=\frac{1}{4 \pi}\left(F_{k i} F_{j}^{k}-\frac{1}{4} g_{i j} F_{k l} F^{k l}\right)
$$

where, using Lorenz gauge, the electromagnetic field tensor $F_{i j}$ can be written in terms of a potential $\left(A_{i}\right)=\left(0,0, A_{\varphi}, A_{t}\right)$,

$$
F_{i j}=A_{i, j}-A_{j, i} .
$$

Note that, like the metric quantities, $A_{\varphi}$ and $A_{t}$ also depend on $R$ and $\theta$ only.

\footnotetext{
${ }^{2}$ For a stationary spacetime, the immediate vicinity of a black hole event horizon must be electrovacuum, see [5 and 2].

3 In the special case without any exterior matter, i.e. for the Kerr-Newman black hole, we obtain BoyerLindquist coordinates $(r, \theta, \varphi, t)$ through a linear transformation $r=2 R+M$, where $M$ is the black hole mass.
} 
In the Boyer-Lindquist-type coordinates, the Einstein-Maxwell equations in electrovacuum are given by ${ }^{4}$

$$
\begin{aligned}
& \left(R^{2}-r_{\mathrm{h}}^{2}\right) \tilde{u}_{, R R}+2 R \tilde{u}_{, R}+\tilde{u}_{, \theta \theta}+\cot \theta \tilde{u}_{, \theta} \\
& =1-\frac{\hat{u}^{2}}{8} \sin ^{2} \theta\left(\omega_{, R}^{2}+\frac{\omega_{, \theta}^{2}}{R^{2}-r_{\mathrm{h}}^{2}}\right)-\frac{1}{\hat{u} \sin ^{2} \theta}\left[\left(R^{2}-r_{\mathrm{h}}^{2}\right) A_{\varphi, R}^{2}+A_{\varphi, \theta}^{2}\right] \\
& -\frac{\hat{u}}{4}\left[\left(\Phi_{, R}-A_{\varphi} \omega_{, R}\right)^{2}+\frac{\left(\Phi_{, \theta}-A_{\varphi} \omega_{, \theta}\right)^{2}}{R^{2}-r_{\mathrm{h}}^{2}}\right], \\
& \left(R^{2}-r_{\mathrm{h}}^{2}\right) \tilde{\mu}_{, R R}+R \tilde{\mu}_{, R}+\tilde{\mu}_{, \theta \theta} \\
& =\frac{\hat{u}^{2}}{16} \sin ^{2} \theta\left(\omega_{, R}^{2}+\frac{\omega_{, \theta}^{2}}{R^{2}-r_{\mathrm{h}}^{2}}\right)-\left(R^{2}-r_{\mathrm{h}}^{2}\right) \tilde{u}_{, R}^{2}+R \tilde{u}_{, R}-\tilde{u}_{, \theta}\left(\tilde{u}_{, \theta}+\cot \theta\right) \text {, } \\
& \left(R^{2}-r_{\mathrm{h}}^{2}\right)\left(\omega_{, R R}+4 \omega_{, R} \tilde{u}_{, R}\right)+\omega_{, \theta \theta}+\omega_{, \theta}\left(3 \cot \theta+4 \tilde{u}_{, \theta}\right) \\
& =\frac{4}{\hat{u} \sin ^{2} \theta}\left[\left(R^{2}-r_{\mathrm{h}}^{2}\right) A_{\varphi, R}\left(\Phi_{, R}-A_{\varphi} \omega_{, R}\right)+A_{\varphi, \theta}\left(\Phi_{, \theta}-A_{\varphi} \omega_{, \theta}\right)\right] \text {, } \\
& \left(R^{2}-r_{\mathrm{h}}^{2}\right)\left[\Phi_{, R R}-A_{\varphi} \omega_{, R R}+2 \tilde{u}_{, R}\left(\Phi_{, R}-A_{\varphi} \omega_{, R}\right)-A_{\varphi, R} \omega_{, R}\right] \\
& +\Phi_{, \theta \theta}-A_{\varphi} \omega_{, \theta \theta}+\left(2 \tilde{u}_{, \theta}+\cot \theta\right)\left(\Phi_{, \theta}-A_{\varphi} \omega_{, \theta}\right)-A_{\varphi, \theta} \omega_{, \theta}=0, \\
& \left(R^{2}-r_{\mathrm{h}}^{2}\right)\left[A_{\varphi, R R}-2 \tilde{u}_{, R} A_{, \varphi, R}\right]+2 R A_{\varphi, R}+A_{\varphi, \theta \theta}-\left(2 \tilde{u}_{, \theta}+\cot \theta\right) A_{\varphi, \theta} \\
& =\frac{\hat{u}^{2}}{4} \sin ^{2} \theta\left[\left(\Phi_{, R}-A_{\varphi} \omega_{, R}\right) \omega_{, R}+\frac{\Phi_{, \theta}-A_{\varphi} \omega_{, \theta}}{R^{2}-r_{\mathrm{h}}^{2}} \omega_{, \theta}\right] \text {. }
\end{aligned}
$$

Here, we have used the dimensionless quantities

$$
\tilde{u}:=\frac{1}{2} \ln \frac{\hat{u}}{\hat{u}_{\mathrm{N}}}, \quad \tilde{\mu}:=\frac{1}{2} \ln \frac{\hat{\mu}}{\hat{u}_{\mathrm{N}}},
$$

where $\hat{u}_{\mathrm{N}}$ is the the north pole value of $\hat{u}$,

$$
\hat{u}_{\mathrm{N}}:=\hat{u}\left(R=r_{\mathrm{h}}, \theta=0\right) .
$$

Moreover, we have replaced $A_{t}$ by the comoving electric potential

$$
\Phi=A_{t}+\omega A_{\varphi}
$$

At the horizon, the metric potentials obey the boundary conditions (cf. [2])

$$
\mathcal{H}: \quad \omega=\text { constant }=\omega_{\mathrm{h}}, \quad \frac{2 r_{\mathrm{h}}}{\sqrt{\hat{\mu} \hat{u}}}=\text { constant }=\kappa, \quad \Phi=\text { constant }=\Phi_{\mathrm{h}},
$$

where $\omega_{\mathrm{h}}, \kappa$, and $\Phi_{\mathrm{h}}$ denote the angular velocity of the horizon, the surface gravity, and the value of the comoving electric potential at the horizon, respectively.

\footnotetext{
4 Throughout this paper we consider a vanishing cosmological constant, $\Lambda=0$. (Note that inequality (1) can be violated for $\Lambda \neq 0$. An example is the Kerr-(A)dS family of black holes, see [3].)
} 
On the horizon's north and south pole $\left(R=r_{\mathrm{h}}\right.$ and $\left.\sin \theta=0\right)$, the following regularity conditions hold ${ }^{5}$ :

$$
\begin{aligned}
& \hat{\mu}\left(R=r_{\mathrm{h}}, \theta=0\right)=\hat{u}\left(R=r_{\mathrm{h}}, \theta=0\right) \\
& \quad=\hat{\mu}\left(R=r_{\mathrm{h}}, \theta=\pi\right)=\hat{u}\left(R=r_{\mathrm{h}}, \theta=\pi\right)=\hat{u}_{\mathrm{N}}=\frac{2 r_{\mathrm{h}}}{\kappa}, \\
& \quad A_{\varphi}(R, \theta=0)=A_{\varphi}(R, \theta=\pi)=0 .
\end{aligned}
$$

In the forthcoming calculations we need relations between metric and electromagnetic quantities at the black hole horizon $\mathcal{H}$. These are provided by an investigation of Eqs. (6) and (7). For the evaluation of these equations in the limit $R \rightarrow r_{\mathrm{h}}$, we introduce the regular horizon potentials (cf. [1])

$$
\hat{\omega}:=\frac{\omega-\omega_{\mathrm{h}}}{R-r_{\mathrm{h}}}, \quad \hat{\Phi}:=\frac{\Phi-\Phi_{\mathrm{h}}}{R-r_{\mathrm{h}}},
$$

from which it follows that

$$
\begin{aligned}
\lim _{R \rightarrow r_{\mathrm{h}}} \frac{\omega_{, \theta}^{2}}{R^{2}-r_{\mathrm{h}}^{2}} & =\lim _{R \rightarrow r_{\mathrm{h}}}\left(\frac{R-r_{\mathrm{h}}}{R+r_{\mathrm{h}}} \hat{\omega}_{, \theta}^{2}\right)=0, \\
\lim _{R \rightarrow r_{\mathrm{h}}} \frac{\left(\Phi_{, \theta}-A_{\varphi} \omega_{, \theta}\right)^{2}}{R^{2}-r_{\mathrm{h}}^{2}} & =\lim _{R \rightarrow r_{\mathrm{h}}}\left[\frac{R-r_{\mathrm{h}}}{R+r_{\mathrm{h}}}\left(\hat{\Phi}_{, \theta}^{2}-A_{\varphi} \hat{\omega}_{, \theta}\right)^{2}\right]=0 .
\end{aligned}
$$

Using these relations, we obtain for (6) and (7) in the limit $R \rightarrow r_{\mathrm{h}}$,

$$
\begin{aligned}
2 r_{\mathrm{h}} \tilde{u}_{, R}+\tilde{u}_{, \theta \theta}+\cot \theta \tilde{u}_{, \theta} & =1-\frac{\hat{u}^{2}}{8} \sin ^{2} \theta \omega_{, R}^{2}-\frac{A_{\varphi, R}^{2}}{\hat{u} \sin ^{2} \theta}-\frac{\hat{u}}{4}\left(\Phi_{, R}-A_{\varphi} \omega_{, R}\right)^{2}, \\
r_{\mathrm{h}} \tilde{\mu}_{, R}+\tilde{\mu}_{, \theta \theta} & =\frac{\hat{u}^{2}}{16} \sin ^{2} \theta \omega_{, R}^{2}+r_{\mathrm{h}} \tilde{u}_{, R}-\tilde{u}_{, \theta}\left(\tilde{u}_{, \theta}+\cot \theta\right) .
\end{aligned}
$$

\section{Calculation of $p_{J}$ and $p_{Q}$}

In order to find suitable expressions for $p_{J}$ and $p_{Q}$, we introduce the following functions which are defined as follows in terms of the metric and electromagnetic quantities at the black hole horizon $\mathcal{H}$ :

$$
\begin{aligned}
U(x) & :=\left.\frac{1}{2} \ln \frac{\hat{u}}{\hat{u}_{\mathrm{N}}}\right|_{\mathcal{H}}, \quad V(x):=\left.\frac{1}{4} \hat{u} \omega_{, R}\right|_{\mathcal{H}}, \\
S(x) & :=\left.\frac{\hat{u}}{2 \sqrt{\hat{u}_{\mathrm{N}}}}\left(\Phi, R-A_{\varphi} \omega_{, R}\right)\right|_{\mathcal{H}}, \quad T(x):=\left.\frac{A_{\varphi}}{\sqrt{\hat{u}_{\mathrm{N}}}}\right|_{\mathcal{H}},
\end{aligned}
$$

where $x:=\cos \theta$.

\footnotetext{
5 Note that (13) holds on the entire rotation axis.
} 
In terms of these quantities we obtain for angular momentum $J$, charge $Q$ and horizon area $A$ (cf. [1]): ${ }^{6}$

$$
\begin{aligned}
J & =\frac{1}{8 \pi} \oint_{\mathcal{H}}\left(m^{i ; j}+2 m^{k} A_{k} F^{i j}\right) \mathrm{d} S_{i j} \\
& =-\left.\frac{1}{4} \int_{0}^{\pi} \hat{u}\left[\frac{\hat{u}}{4} \omega_{, R} \sin ^{2} \theta-A_{\varphi}\left(\Phi_{, R}-A_{\varphi} \omega_{, R}\right)\right]\right|_{\mathcal{H}} \sin \theta \mathrm{d} \theta \\
& =-\frac{\hat{u}_{\mathrm{N}}}{4} \int_{-1}^{1}\left[V \mathrm{e}^{2 U}\left(1-x^{2}\right)-2 S T\right] \mathrm{d} x, \\
Q & =-\frac{1}{4 \pi} \oint_{\mathcal{H}} F^{i j} \mathrm{~d} S_{i j}=-\left.\frac{1}{4} \int_{0}^{\pi} \hat{u}\left(\Phi_{, R}-A_{\varphi} \omega_{, R}\right)\right|_{\mathcal{H}} \sin \theta \mathrm{d} \theta \\
& =-\frac{\sqrt{\hat{u}_{\mathrm{N}}}}{2} \int_{-1}^{1} S \mathrm{~d} x \\
A & =\left.2 \pi \int_{0}^{\pi} \sqrt{\hat{\mu} \hat{u}}\right|_{\mathcal{H}} \sin \theta \mathrm{d} \theta=4 \pi \hat{u}_{\mathrm{N}} .
\end{aligned}
$$

Here, we have used conditions (11) and (12). Finally, we arrive at

$$
\begin{aligned}
& p_{J} \equiv \frac{8 \pi J}{A}=-\frac{1}{2} \int_{-1}^{1}\left[V \mathrm{e}^{2 U}\left(1-x^{2}\right)-2 S T\right] \mathrm{d} x, \\
& p_{Q} \equiv \frac{4 \pi Q^{2}}{A}=\frac{1}{4}\left(\int_{-1}^{1} S \mathrm{~d} x\right)^{2} .
\end{aligned}
$$

\section{Reformulation in terms of a Variational Problem}

As a first step towards the proof of the inequality (2) for sub-extremal black holes, we consider the following lemma.

Lemma 1 (Characterization of sub-extremal black holes). A necessary condition for the existence of trapped surfaces in the interior vicinity of the event horizon of an axisymmetric and stationary charged black hole is

$$
\left.\int_{0}^{\pi}(\hat{\mu} \hat{u})_{, R}\right|_{\mathcal{H}} \sin \theta \mathrm{d} \theta>0 .
$$

This lemma was originally derived in the setting of pure Einsteinian gravity (without Maxwell-field), see [7]. As the corresponding proof presented in [7] carries over to the full Einstein-Maxwell theory, we may use the lemma in the forthcoming investigation.

The proof of (2) relies on showing that for $p_{J}^{2}+p_{Q}^{2} \geq 1$ inequality (25) is violated, which implies by virtue of Lemma 1 a violation of the sub-extremality condition:

$$
p_{J}^{2}+p_{Q}^{2} \geq\left. 1 \quad \Rightarrow \quad \int_{0}^{\pi}(\hat{\mu} \hat{u})_{, R}\right|_{\mathcal{H}} \sin \theta \mathrm{d} \theta \leq 0 .
$$

\footnotetext{
${ }^{6}$ Note that $m^{i}$ denotes the Killing vector with respect to axisymmetry.
} 
Using Einstein equations (15) and (16) together with the boundary conditions (11), we may rewrite the integrand in (25) as

$$
\begin{aligned}
\left.(\hat{\mu} \hat{u})_{, R}\right|_{\mathcal{H}}= & \frac{2\left(\hat{u}_{\mathrm{N}}\right)^{2}}{r_{\mathrm{h}}}\left[1-\left(\frac{\hat{u} \omega_{, R}}{4}\right)^{2} \sin ^{2} \theta-\tilde{u}_{, \theta}\left(\tilde{u}_{, \theta}+2 \cot \theta\right)\right. \\
& \left.-\frac{A_{\varphi, \theta}^{2}}{\hat{u} \sin ^{2} \theta}-\frac{\hat{u}}{4}\left(\Phi_{, R}-A_{\varphi} \omega_{, R}\right)^{2}\right]
\end{aligned}
$$

Hence we can express (25) in terms of $S, T, U$, and $V$ :

$$
\frac{1}{2} \int_{-1}^{1}\left[\left(V^{2}+U^{\prime 2}\right)\left(1-x^{2}\right)-2 x U^{\prime}+\left(S^{2}+T^{\prime 2}\right) \mathrm{e}^{-2 U}\right] \mathrm{d} x<1,
$$

where $^{\prime}:=\mathrm{d} / \mathrm{d} x$. With the expressions for $p_{J}$ and $p_{Q}$ [see (23), (24)] we can thus write the implication in (26) as follows:

$$
\begin{aligned}
& \left(\int_{-1}^{1}\left[V \mathrm{e}^{2 U}\left(1-x^{2}\right)-2 S T\right] \mathrm{d} x\right)^{2}+\frac{1}{4}\left(\int_{-1}^{1} S \mathrm{~d} x\right)^{4} \geq 4 \\
& \Rightarrow \frac{1}{2} \int_{-1}^{1}\left[\left(V^{2}+U^{\prime 2}\right)\left(1-x^{2}\right)-2 x U^{\prime}+\left(S^{2}+T^{\prime 2}\right) \mathrm{e}^{-2 U}\right] \mathrm{d} x \geq 1
\end{aligned}
$$

In the following we show that this implication holds for all sufficiently regular functions ${ }^{7}$

$$
S, T, U, V:[-1,1] \rightarrow \mathbb{R}
$$

which satisfy the boundary conditions

$$
U( \pm 1)=T( \pm 1)=0 .
$$

The conditions in (30) follow from (17), (18), (12), (13).

In the next step we formulate a variational problem which is a sufficient criterion for the validity of the implication in (29). Applying the Cauchy-Schwarz inequality to the first inequality in (29), we obtain

$$
\left(\sqrt{\int_{-1}^{1} V^{2}\left(1-x^{2}\right) \mathrm{d} x} \sqrt{\int_{-1}^{1} \mathrm{e}^{4 U}\left(1-x^{2}\right) \mathrm{d} x}+2\left|\int_{-1}^{1} S T \mathrm{~d} x\right|\right)^{2}+\frac{1}{4}\left(\int_{-1}^{1} S \mathrm{~d} x\right)^{4} \geq 4 .
$$

With the abbreviations

$$
c_{1}:=\sqrt{\int_{-1}^{1} \mathrm{e}^{4 U}\left(1-x^{2}\right) \mathrm{d} x}, \quad c_{2}:=\int_{-1}^{1} S T \mathrm{~d} x, \quad c_{3}:=\frac{1}{\sqrt{2}} \int_{-1}^{1} S \mathrm{~d} x,
$$

this inequality leads to the estimate

$$
\int_{-1}^{1} V^{2}\left(1-x^{2}\right) \mathrm{d} x \geq M_{2}^{2}
$$

\footnotetext{
7 A precise statement about the required regularity properties follows below.
} 
where

$$
M_{1}:=\max \left\{0,4-c_{3}^{4}\right\}, \quad M_{2}:=\max \left\{0, \frac{\sqrt{M_{1}}-2\left|c_{2}\right|}{c_{1}}\right\} .
$$

Using (32) in order to replace the term $\int V^{2}\left(1-x^{2}\right) \mathrm{d} x$ in the second inequality in (29), it follows immediately that

$$
I[S, T, U]:=\frac{1}{2} \int_{-1}^{1}\left[U^{\prime 2}\left(1-x^{2}\right)-2 x U^{\prime}(x)+\left(S^{2}+T^{\prime 2}\right) \mathrm{e}^{-2 U}\right] \mathrm{d} x+\frac{M_{2}^{2}}{2} \geq 1
$$

is a sufficient condition for the validity of the implication in (26). We summarize this result in the following lemma.

Lemma 2 (Variational problem). The inequality $p_{J}^{2}+p_{Q}^{2}<1$ holds for any sub-extremal axisymmetric and stationary charged black hole with surrounding matter provided that the inequality

$$
I[S, T, U] \geq 1
$$

is satisfied for all $S \in L^{2}(-1,1), T, U \in W_{0}^{1,2}(-1,1)$.

Remark. The Lebesgue and Sobolev spaces $L^{2}$ and $W_{0}^{1,2}$ contain all functions $S$ and $T$, $U$, respectively, that arise in the physical situation above.

With this lemma, we have reduced inequality (2) to the variational problem of calculating the minimum of $I[S, T, U]$ and showing that this is greater than or equal to 1 . In the next section, we solve this problem with methods from the calculus of variations.

\section{Solution of the Variational Problem}

5.1. An approximating family of functionals. Analyzing the functional $I$ proves difficult as the factor $1-x^{2}$ is singular at the boundary $x= \pm 1$, cf. definition of $I$ in (34). We therefore approximate it by a family of slightly modified functionals $I_{\varepsilon}$ which are conducive to analysis using techniques of the calculus of variations. We work on the Hilbert space

$$
X:=\left(L^{2} \times W_{0}^{1,2} \times W_{0}^{1,2}\right)(-1,1)
$$

endowed with the inner product

$$
\langle(S, T, U),(\tilde{S}, \tilde{T}, \tilde{U})\rangle:=\int_{-1}^{1}\left[S \tilde{S}+T^{\prime} \tilde{T}^{\prime}+U^{\prime} \tilde{U}^{\prime}\left(1+\varepsilon-x^{2}\right)\right] \mathrm{d} x
$$

depending on a fixed $\varepsilon>0$. Recall that this inner product is equivalent to the ordinary one by the fundamental theorem of calculus. Moreover, we have

Proposition 1 (Theorem 2.2 in Buttazzo-Giaquinta-Hildebrandt [4]). On any bounded interval $J \subseteq \mathbb{R}, W^{1,2}(J) \hookrightarrow C^{0}(\bar{J})$ compactly. Moreover, the fundamental theorem of calculus holds in $W^{1,2}(J)$. 
For $\varepsilon \geq 0$, we consider the functional $I_{\varepsilon}: X \rightarrow \mathbb{R}$ given by $I_{\varepsilon}[S, T, U]:=\frac{1}{2} \int_{-1}^{1}\left[U^{\prime 2}\left(1+\varepsilon-x^{2}\right)-2 x U^{\prime}+\left(S^{2}+T^{\prime 2}\right) \mathrm{e}^{-2 U}\right] \mathrm{d} x+\frac{M_{2}^{\varepsilon}[S, T, U]^{2}}{2}$,

where the auxiliary functionals $M_{2}^{\varepsilon}, M_{1}, c_{1}^{\varepsilon}, c_{2}, c_{3}: X \rightarrow \mathbb{R}$ are defined by

$$
\begin{aligned}
& c_{1}^{\varepsilon}[S, T, U]:=\sqrt{\int_{-1}^{1} \mathrm{e}^{4 U}\left(1+\varepsilon-x^{2}\right) \mathrm{d} x}, \quad c_{2}[S, T, U]:=\int_{-1}^{1} S T \mathrm{~d} x, \\
& c_{3}[S, T, U]:=\frac{1}{\sqrt{2}} \int_{-1}^{1} S \mathrm{~d} x, \quad M_{1}[S, T, U]:=\max \left\{0,4-\left(c_{3}[S, T, U]\right)^{4}\right\},
\end{aligned}
$$

and

$$
M_{2}^{\varepsilon}[S, T, U]:=\max \left\{0,\left(\frac{\sqrt{M_{1}}-2\left|c_{2}\right|}{c_{1}^{\varepsilon}}\right)[S, T, U]\right\},
$$

respectively. All of these functionals can easily be seen to be well-defined, and all auxiliary functionals are weakly continuous on $X$ by Poincaré's inequality. Also, $c_{1}^{\varepsilon}$ is positive and both $M_{1}, M_{2}^{\varepsilon}$ are non-negative.

We now show that for $\varepsilon>0$ there exists a global minimizer $(S, T, U) \in X$ for $I_{\varepsilon}$ and study its value $I_{\varepsilon}[S, T, U]$. Following this investigation, we take the limit $\varepsilon \rightarrow 0$ and see that the claim of Lemma 2 follows.

5.2. Existence and characterization of the minimizer. Now let $\varepsilon>0$ be fixed. $I_{\varepsilon}$ then has the following properties:

(i) $I_{\varepsilon}$ is bounded from below. Using $0 \leq\left(\frac{x}{\sqrt{1+\varepsilon-x^{2}}}-U^{\prime}(x) \sqrt{1+\varepsilon-x^{2}}\right)^{2}=$ $\frac{x^{2}}{1+\varepsilon-x^{2}}-2 x U^{\prime}(x)+U^{\prime 2}(x)\left(1+\varepsilon-x^{2}\right)$ we conclude that

$$
I_{\varepsilon}[S, T, U] \geq-\frac{1}{2} \int_{-1}^{1} \frac{x^{2}}{1+\varepsilon-x^{2}} \mathrm{~d} x=: C(\varepsilon)>-\infty
$$

for any $(S, T, U) \in X$.

(ii) $I_{\varepsilon}$ is coercive with respect to the weak topology on $X$. Indeed, applying the Cauchy-Schwarz inequality to $\int_{-1}^{1} x U^{\prime}(x) \mathrm{d} x$, we obtain that

$$
I_{\varepsilon}[S, T, U] \geq \frac{1}{2}\|U\|^{2}-C(\varepsilon)\|U\|
$$

for any $(S, T, U) \in X$ with $C(\varepsilon)>0$. Hence, for every $P \in \mathbb{R}$ there exists $Q_{P} \in \mathbb{R}$ such that $I_{\varepsilon}[S, T, U] \geq P$ whenever $\|(S, T, U)\| \geq Q_{P}$. This is equivalent to coercivity of the functional $I_{\varepsilon}$ with respect to the weak topology on $X$, where both the norm $\|\cdot\|$ and the weak topology refer to the inner product defined above. 
(iii) The functional $I_{\varepsilon}$ is sequentially lower semi-continuous (lsc) with respect to the weak topology on $X$. To see this, recall that lower semi-continuity is additive and that the first terms can be dealt with by standard theory (see e.g. [9]), and use Proposition 1 as well as the Lipschitz continuity of exp on bounded intervals. For the last term, the weak continuity of the auxiliary functionals yields the claim.

We are now in a position to show existence of a global minimizer for $I_{\varepsilon}$ : As we have seen in (i), $I_{\varepsilon}$ is bounded from below on $X$. We can hence choose a minimizing sequence $\left(S_{k}, T_{k}, U_{k}\right) \in X$ which must be bounded by coercivity (ii) and thus has a weakly converging subsequence by Hilbert space techniques (theorem of EberleinShmulyan [9]) tending to a limit $\left(S^{*}, T^{*}, U^{*}\right)$. Lower semicontinuity as in (iii) then gives us $I_{\varepsilon}\left[S^{*}, T^{*}, U^{*}\right]=\inf \left\{I_{\varepsilon}[S, T, U] \mid(S, T, U) \in X\right\}$ and thus asserts that $\left(S^{*}, T^{*}, U^{*}\right)$ is a global minimizer. However, $I_{\varepsilon}$ is not Fréchet-differentiable at $(S, T, U) \in X$ with $c_{2}[S, T, U]=0$ and $c_{3}^{4}[S, T, U]=4$ due to the maximum-terms in the definitions of $M_{i}^{\varepsilon}(i=1,2)$. It is consequently impossible to derive Euler-Lagrange equations for $I_{\varepsilon}$ directly.

To circumvent this problem, we introduce the constraints $c_{i}^{\varepsilon}=$ constant $(i=1,2,3)$ and use the method of Lagrange multipliers to minimize $I_{\varepsilon}$ under these constraints. This leads to a Fréchet-differentiable functional on every class $\mathcal{K}$ with fixed values of $c_{i}^{\varepsilon}$. Moreover, the asserted global minimizer $\left(S^{*}, T^{*}, U^{*}\right)$ also minimizes $I_{\varepsilon}$ in its class $\mathcal{K}^{*}$ which induces conditions on the constants specifying $\mathcal{K}^{*}$ and explicit expressions for the related Lagrange multipliers.

\subsection{The Euler-Lagrange equations. Setting}

$$
c_{i}^{*}:=c_{i}^{\varepsilon}\left[S^{*}, T^{*}, U^{*}\right] \text { and } M_{j}^{*}:=M_{j}^{\varepsilon}\left[S^{*}, T^{*}, U^{*}\right], \quad(i=1,2,3 ; j=1,2),
$$

the class $\mathcal{K}^{*}$ containing the global minimizer $\left(S^{*}, T^{*}, U^{*}\right)$ is characterized by

$$
\mathcal{K}^{*}:=\left\{(S, T, U) \in X \mid c_{i}^{\varepsilon}[S, T, U]=c_{i}^{*}(i=1,2,3)\right\} .
$$

In this class, $I_{\varepsilon}$ can be evaluated as follows:

$$
I_{\varepsilon}[S, T, U]=\frac{1}{2} \int_{-1}^{1}\left[U^{\prime 2}\left(1+\varepsilon-x^{2}\right)-2 x U^{\prime}+\left(S^{2}+T^{\prime 2}\right) \mathrm{e}^{-2 U}\right] \mathrm{d} x+\frac{\left(M_{2}^{*}\right)^{2}}{2}
$$

By the theory of Lagrange multipliers, for each minimizer $(S, T, U)$ of $I_{\varepsilon}$ in the class $\mathcal{K}^{*}$, there is $\left(\lambda_{1}, \lambda_{2}, \lambda_{3}\right) \in \mathbb{R}^{3}$ such that $\left(S, T, U, \lambda_{1}, \lambda_{2}, \lambda_{3}\right) \in X \times \mathbb{R}^{3}$ is a critical point of the functional $J_{\varepsilon}^{*}: X \times \mathbb{R}^{3} \rightarrow \mathbb{R}$ given by

$$
\begin{aligned}
J_{\varepsilon}^{*}\left[S, T, U, \lambda_{1}, \lambda_{2}, \lambda_{3}\right]:= & \frac{1}{2} \int_{-1}^{1}\left[U^{\prime 2}\left(1+\varepsilon-x^{2}\right)-2 x U^{\prime}+\left(S^{2}+T^{\prime 2}\right) \mathrm{e}^{-2 U}\right] \mathrm{d} x \\
& +\lambda_{1}\left(\left(c_{1}^{\varepsilon}[S, T, U]\right)^{2}-\left(c_{1}^{*}\right)^{2}\right)+\lambda_{2}\left(c_{2}[S, T, U]-c_{2}^{*}\right) \\
& +\sqrt{2} \lambda_{3}\left(c_{3}[S, T, U]-c_{3}^{*}\right),
\end{aligned}
$$

which is well-defined and indeed sufficiently smooth by Proposition 1 . In other words, there is $\left(\lambda_{1}^{*}, \lambda_{2}^{*}, \lambda_{3}^{*}\right) \in \mathbb{R}^{3}$ such that $\left(S^{*}, T^{*}, U^{*}\right)$ satisfies

$$
\begin{aligned}
0= & \int_{-1}^{1}\left[U^{\prime} \psi^{\prime}\left(1+\varepsilon-x^{2}\right)-x \psi^{\prime}\right] \mathrm{d} x+\int_{-1}^{1}\left[S \rho+T^{\prime} \varphi^{\prime}-\left(S^{2}+T^{\prime 2}\right) \psi\right] \mathrm{e}^{-2 U} \mathrm{~d} x \\
& +4 \lambda_{1}^{*} \int_{-1}^{1} \mathrm{e}^{4 U} \psi\left(1+\varepsilon-x^{2}\right) \mathrm{d} x+\lambda_{2}^{*} \int_{-1}^{1}(S \varphi+T \rho) \mathrm{d} x+\lambda_{3}^{*} \int_{-1}^{1} \rho \mathrm{d} x
\end{aligned}
$$


for all $(\rho, \varphi, \psi) \in X$. This can be restated by saying that $\left(S^{*}, T^{*}, U^{*}\right)$ is a weak solution of

$$
\begin{aligned}
& 0=-U^{\prime \prime}\left(1+\varepsilon-x^{2}\right)+2 x U^{\prime}+1-\left(S^{2}+T^{\prime 2}\right) \mathrm{e}^{-2 U}+4 \lambda_{1}^{*}\left(1+\varepsilon-x^{2}\right) \mathrm{e}^{4 U}, \\
& 0=-T^{\prime \prime}+2 U^{\prime} T^{\prime}+\lambda_{2}^{*} S \mathrm{e}^{2 U} \\
& 0=S+\left(\lambda_{2}^{*} T+\lambda_{3}^{*}\right) \mathrm{e}^{2 U} \\
& 0=T( \pm 1)=U( \pm 1)
\end{aligned}
$$

on $(-1,1)$. Any weak solution $(S, T, U) \in X$ of the system (42), (43), and (44) can be shown to be smooth and to satisfy the equations strongly via a bootstrap argument: For all $(\rho, \varphi, \psi) \in X$, we can rewrite (41) as

$$
\begin{aligned}
0= & \int_{-1}^{1}\left[U^{\prime}\left(1+\varepsilon-x^{2}\right)-x+\int_{-1}^{x}\left(S^{2}+T^{\prime 2}\right) \mathrm{e}^{-2 U} \mathrm{~d} t\right. \\
& \left.-4 \lambda_{1}^{*} \int_{-1}^{x} \mathrm{e}^{4 U}\left(1+\varepsilon-t^{2}\right) \mathrm{d} t\right] \psi^{\prime} \mathrm{d} x, \\
0= & \int_{-1}^{1}\left[T^{\prime} \mathrm{e}^{-2 U}-\lambda_{2}^{*} \int_{-1}^{x} S \mathrm{~d} t\right] \varphi^{\prime} \mathrm{d} x, \\
0= & \int_{-1}^{1}\left[S \mathrm{e}^{-2 U}+\lambda_{2}^{*} T+\lambda_{3}^{*}\right] \rho \mathrm{d} x,
\end{aligned}
$$

where we used integration by parts and Proposition 1. By the fundamental lemma of the calculus of variations, there are constants $a, b \in \mathbb{R}$ such that the equations

$$
\begin{aligned}
a= & U^{\prime}(x)\left(1+\varepsilon-x^{2}\right)-x+\int_{-1}^{x}\left(S^{2}+T^{\prime 2}\right) \mathrm{e}^{-2 U} \mathrm{~d} t \\
& -4 \lambda_{1}^{*} \int_{-1}^{x} \mathrm{e}^{4 U}\left(1+\varepsilon-t^{2}\right) \mathrm{d} t \\
b= & T^{\prime}(x) \mathrm{e}^{-2 U(x)}-\lambda_{2}^{*} \int_{-1}^{x} S \mathrm{~d} t \\
0= & S(x) \mathrm{e}^{-2 U(x)}+\lambda_{2}^{*} T(x)+\lambda_{3}^{*}
\end{aligned}
$$

hold almost everywhere on $(-1,1)$. Solving iteratively for $T^{\prime}, U^{\prime}$, and $S$, we deduce the respective smoothness of $S, T$, and $U$ up to the boundary by a bootstrap argument (similar to p. 462 in [6]) using Propostion 1 in every step. Differentiating Eqs. (45) and (46), we get validity of (42) and (43) in the strong sense. In particular, $\left(S^{*}, T^{*}, U^{*}\right)$ is a smooth classical solution of the Euler-Lagrange equations of $J_{\varepsilon}^{*}$ with $\left(\lambda_{1}, \lambda_{2}, \lambda_{3}\right)=\left(\lambda_{1}^{*}, \lambda_{2}^{*}, \lambda_{3}^{*}\right)$.

5.4. Solution of the Euler-Lagrange equations. Let us now determine the minimizer $(S, T, U):=\left(S^{*}, T^{*}, U^{*}\right)$ explicitly, dropping the asterisk in what follows for ease of notation. $S$ can obviously be expressed as

$$
S(x)=-\left[\lambda_{3}+\lambda_{2} T(x)\right] \mathrm{e}^{2 U(x)}
$$

by Eq. (44). Inserting this expression into (43), we get the equation

$$
0=T^{\prime \prime}-2 U^{\prime} T^{\prime}+\lambda_{2}\left(\lambda_{3}+\lambda_{2} T\right) \mathrm{e}^{4 U},
$$


a linear ODE of second order for $T$ for given $U$. To solve (49), consider two separate cases:

(i) Assume $\lambda_{2}=0$. Then (49) reduces to $T^{\prime \prime}=2 U T^{\prime}$ which has the general solution $T(x)=a \int_{-1}^{x} \mathrm{e}^{2 U(t)} \mathrm{d} t+b$ with $a, b \in \mathbb{R}$, so that $T( \pm 1)=0$ induces $T \equiv 0$.

(ii) Assume now $\lambda_{2} \neq 0$. In this case, (49) has the general solution

$$
T(x)=\frac{\lambda_{3}}{\lambda_{2}}\left[a \sin \left(\lambda_{2} \int_{-1}^{x} \mathrm{e}^{2 U(t)} \mathrm{d} t\right)+b \cos \left(\lambda_{2} \int_{-1}^{x} \mathrm{e}^{2 U(t)} \mathrm{d} t\right)-1\right]
$$

with $a, b \in \mathbb{R}$. For $\lambda_{3} \neq 0$, inserting the boundary values $T( \pm 1)=0$ gives us $b=1$ and

$$
a=\frac{1-\cos \left(\lambda_{2} \int_{-1}^{1} \mathrm{e}^{2 U(t)} \mathrm{d} t\right)}{\sin \left(\lambda_{2} \int_{-1}^{1} \mathrm{e}^{2 U(t)} \mathrm{d} t\right)}= \pm \sqrt{\frac{1-\cos \left(\lambda_{2} \int_{-1}^{1} \mathrm{e}^{2 U(t)} \mathrm{d} t\right)}{1+\cos \left(\lambda_{2} \int_{-1}^{1} \mathrm{e}^{2 U(t)} \mathrm{d} t\right)}} .
$$

The task of determining $U$ remains to be completed. To this end, set

$$
\gamma:=-\left[S(x)^{2}+T^{\prime}(x)^{2}\right] \mathrm{e}^{-4 U(x)} \leq 0
$$

and observe that $\mathrm{d} \gamma / \mathrm{d} x=0$, so that $\gamma$ is a non-positive constant. Moreover, from the explicit expressions obtained for $S$ and $T$, we see that $\gamma=-\lambda_{3}^{2}\left(1+a^{2}\right)$ where, as defined above, $a=0$ if $\lambda_{2}=0$ and $a$ is as in (51) otherwise.

Recall that $(S, T, U)$ is a global minimizer of $I_{\varepsilon}$. Although $I_{\varepsilon}$ is not globally Fréchetdifferentiable w.r.t. $S$ and $T$, it can straightforwardly be shown that it is continuously Fréchet-differentiable w.r.t. $U$. We thus deduce via integration by parts and by the fundamental lemma of the calculus of variations that

$$
0=-U^{\prime \prime}\left(1+\varepsilon-x^{2}\right)+2 x U^{\prime}+1-\left(S^{2}+T^{\prime 2}\right) \mathrm{e}^{-2 U}-\frac{2 M_{2}^{2}}{c_{1}^{2}}\left(1+\varepsilon-x^{2}\right) \mathrm{e}^{4 U} .
$$

Comparing this equation with (42), we obtain the explicit expression

$$
\lambda_{1}=-\frac{M_{2}^{2}}{2 c_{1}^{2}} \leq 0 .
$$

Moreover, the Euler-Lagrange equation (42) for $U$, which can now be written as

$$
0=-U^{\prime \prime}\left(1+\varepsilon-x^{2}\right)+2 x U^{\prime}+1+\gamma \mathrm{e}^{2 U}+4 \lambda_{1}\left(1+\varepsilon-x^{2}\right) \mathrm{e}^{4 U},
$$

has an integrating factor and leads to the first order ODE

$$
\begin{aligned}
F:= & -\left(1+\varepsilon-x^{2}\right)^{2} U^{\prime 2}+2 x\left(1+\varepsilon-x^{2}\right) U^{\prime}+2 \lambda_{1} \mathrm{e}^{4 U}\left(1+\varepsilon-x^{2}\right)^{2} \\
& -x^{2}+\gamma\left(1+\varepsilon-x^{2}\right) \mathrm{e}^{2 U} \equiv \text { constant }
\end{aligned}
$$

because

$$
\begin{aligned}
F^{\prime}(x)= & 2\left[\left(1-x^{2}\right) U^{\prime}(x)-x\right]\left[-U^{\prime \prime}\left(1+\varepsilon-x^{2}\right)+2 x U^{\prime}+1+\gamma \mathrm{e}^{2 U}\right. \\
& \left.+4 \lambda_{1}\left(1+\varepsilon-x^{2}\right) \mathrm{e}^{4 U}\right]=0 .
\end{aligned}
$$


We now proceed to calculate $U$. Substituting $W(x):=\left(1+\varepsilon-x^{2}\right) \mathrm{e}^{2 U(x)}>0$ on $[-1,1]$, Eq. (54) can be reformulated to say

$$
\frac{W^{\prime}}{2 W}= \pm \frac{\sqrt{2 \lambda_{1} W^{2}+\gamma W-F}}{1+\varepsilon-x^{2}}
$$

which in particular implies $F \leq 0$ as both $\lambda_{1}, \gamma \leq 0$ and $W>0$ by definition. We would like to divide by the square root on the right hand side and integrate the equation. We must first find out where the zeros of $W^{\prime}$ can lie, if they exist at all. A careful discussion of the ODE (55) referring to (42), the boundary values $W( \pm 1)=\varepsilon$, and using the fact that we are discussing the class $\mathcal{K}^{*}$ containing the global minimizer, shows that $W^{\prime}$ has exactly one zero $\tilde{x} \in(-1,1)$ and that $W^{\prime \prime}(\tilde{x})<0$. Moreover, from this discussion we obtain $F<0$ and the fact that $\lambda_{1}$ and $\gamma$ cannot vanish simultaneously.

Integrating (55) on both $[-1, \tilde{x})$ and $(\tilde{x}, 1]$ and using $W^{\prime \prime}(\tilde{x})<0$ to determine the correct sign on each interval we obtain

$$
W_{ \pm}(x)=\frac{2 F}{\gamma-\sqrt{\gamma^{2}+8 \lambda_{1} F} \cosh y_{ \pm}(x)}, \quad y_{ \pm}(x):=\frac{2 \sqrt{-F}}{\sqrt{1+\varepsilon}} \operatorname{artanh}\left(\frac{x}{\sqrt{1+\varepsilon}}\right) \pm C,
$$

where

$$
\begin{gathered}
C=-\frac{2 \sqrt{-F}}{\sqrt{1+\varepsilon}} \operatorname{artanh}\left(\frac{1}{\sqrt{1+\varepsilon}}\right)+\operatorname{artanh}\left(\frac{\sqrt{2 \lambda_{1} \varepsilon^{2}+\gamma \varepsilon-F}}{\sqrt{-F}+\frac{\gamma \varepsilon}{2 \sqrt{-F}}}\right), \\
W_{-}:[-1, \tilde{x}) \rightarrow \mathbb{R}, \quad W_{+}:(\tilde{x}, 1] \rightarrow \mathbb{R} .
\end{gathered}
$$

As the solution $W$ we are looking for is smooth by the above and agrees with $W_{ \pm}$where they exist, $W_{-}$and $W_{+}$must smoothly fit together at $\tilde{x}$. Also, the induced functions $U_{-}, U_{+}$both smoothly extend to $[-1,1]$ and must agree at $\tilde{x}$ to all orders. Moreover, they both solve Eq. (42). Thus, Picard's uniqueness theorem (cf. p. 9 in [8]) tells us they agree on the whole interval $[-1,1]$. From $W_{-}(-x)=W_{+}(x)$ we deduce symmetry of $W, \tilde{x}=0$, and $C=0$.

Altogether, we know that $W$ has the following form:

$$
W(x)=\frac{2 F}{\gamma-\sqrt{\gamma^{2}+8 \lambda_{1} F} \cosh y(x)}, \quad y(x):=\frac{2 \sqrt{-F}}{\sqrt{1+\varepsilon}} \operatorname{artanh}\left(\frac{x}{\sqrt{1+\varepsilon}}\right) .
$$

5.5. Estimating the minimal value of $I_{\varepsilon}$. In order to estimate the value of $I_{\varepsilon}$ at its global minimizer, we use the fact that (54) allows us to simplify our expression for $I_{\varepsilon}$. Using (53), we obtain

$$
I_{\varepsilon}[S, T, U]=1-\left(\sqrt{1+\varepsilon}+\frac{F}{\sqrt{1+\varepsilon}}\right) \operatorname{artanh} \frac{1}{\sqrt{1+\varepsilon}} .
$$

We now intend to estimate $F$ from above via

$$
F \leq-(1+\varepsilon)\left[1-\frac{2 \ln \frac{2+\varepsilon}{2-\varepsilon}}{\ln \frac{(2+\varepsilon)^{2}}{\varepsilon}}\right]^{2}
$$

which allows us to conclude that $\liminf _{\varepsilon \rightarrow 0} I_{\varepsilon} \geq 1$, see Subsect. 5.8. We prepare this estimate with the study of two auxiliary functions $f$ and $g$, see below. We then use these 
functions to obtain (58) in the cases $c_{2} \neq 0$ and $c_{2}=0$ (and several subcases), see Subsecs. 5.6 and 5.7.

We define

$$
f(\alpha):=\frac{1}{2} M_{2}^{\varepsilon}[(1+\alpha) S, T, U]^{2}, \quad g(\alpha):=\frac{1}{2} M_{2}^{\varepsilon}[S,(1+\alpha) T, U]^{2} .
$$

The function $g: \mathbb{R} \rightarrow \mathbb{R}$ can be seen to be differentiable at $\alpha=0$ and we obtain

$$
g^{\prime}(0)=-\frac{2\left|c_{2}\right| M_{2}}{c_{1}} .
$$

As $(S, T, U)$ simultaneously is a minimizer of $I_{\varepsilon}$ and a critical point of $J_{\varepsilon}$, it follows from (41) on the other hand that

$$
0=\int_{-1}^{1} T^{\prime 2} \mathrm{e}^{-2 U} \mathrm{~d} x+\lambda_{2} c_{2}=\int_{-1}^{1} T^{\prime 2} \mathrm{e}^{-2 U} \mathrm{~d} x+g^{\prime}(0) .
$$

We also find that $f: \mathbb{R} \rightarrow \mathbb{R}$ is differentiable at $\alpha=0$ unless both $c_{2}=0$ and $c_{3}^{4}=4$. Recall that this singular case also led us to the introduction of Lagrange multipliers. We then have

$$
f^{\prime}(0)=\left\{\begin{array}{ll}
g^{\prime}(0)-\frac{2 M_{2} c_{3}^{4}}{c_{1} \sqrt{M_{1}}} & \text { if } M_{1} \neq 0 \\
0 & \text { if } M_{1}=0
\end{array},\right.
$$

unless both $c_{2}=0$ and $c_{3}^{4}=4$. In addition, it follows as above that

$$
0=\int_{-1}^{1} S^{2} \mathrm{e}^{-2 U} \mathrm{~d} x+\lambda_{2} c_{2}+\sqrt{2} \lambda_{3} c_{3}=\int_{-1}^{1} S^{2} \mathrm{e}^{-2 U} \mathrm{~d} x+f^{\prime}(0),
$$

or equivalently

$$
\begin{aligned}
0 & =-\gamma \int_{-1}^{1} \mathrm{e}^{2 U} \mathrm{~d} x-\int_{-1}^{1} T^{\prime 2} \mathrm{e}^{-2 U} \mathrm{~d} x+\lambda_{2} c_{2}+\sqrt{2} \lambda_{3} c_{3} \\
& =-\gamma \int_{-1}^{1} \mathrm{e}^{2 U} \mathrm{~d} x-\int_{-1}^{1} T^{\prime 2} \mathrm{e}^{-2 U} \mathrm{~d} x+f^{\prime}(0) .
\end{aligned}
$$

5.6. Estimating the minimal value of $I_{\varepsilon}$ : the case $c_{2}=0$. The explicit expression (59) for $g^{\prime}(0)$ suggests separate treatment of the cases $c_{2}=0$ and $c_{2} \neq 0$. We begin with $c_{2}=0$. Four different subcases arise, namely

(a) $c_{3}^{4}=4$,

(b) $c_{3}=0$,

(c) $c_{3} \neq 0, M_{1}=0$,

(d) $c_{3} \neq 0, M_{1} \neq 0$.

We will find that the last two cases cannot occur in the minimizing class $\mathcal{K}^{*}$. In the first two cases, we will indeed arrive at estimate (58).

Let us discuss the singular case (a) first. Here, (60) implies $T \equiv 0, c_{3} \neq 0$ assures $S \neq 0$ so that we can deduce $\lambda_{2}=0$ from (43). Recall $M_{1}=M_{2}=\lambda_{1}=0, \gamma \neq 0$. 
Then (48) implies $S=-\lambda_{3} \mathrm{e}^{2 U}$ so that $c_{3}=-\frac{\lambda_{3}}{\sqrt{2}} \int_{-1}^{1} \mathrm{e}^{2 U} \mathrm{~d} x$. Let us proceed to calculate $\int_{-1}^{1} \mathrm{e}^{2 U} \mathrm{~d} x$. The boundary condition $W( \pm 1)=\varepsilon$ implies $\gamma=\frac{2 F}{\varepsilon\left(1+\cosh y_{1}\right)}$ and we are in a position to calculate

$\int_{-1}^{1} \mathrm{e}^{2 U(x)} \mathrm{d} x=\int_{-1}^{1} \frac{W(x)}{1+\varepsilon-x^{2}} \mathrm{~d} x=-\frac{\sqrt{-F}}{2 \gamma} \int_{-y_{1}}^{y_{1}}\left[1-\tanh ^{2}\left(\frac{y}{2}\right)\right] \mathrm{d} y=\frac{\varepsilon \sinh \left(y_{1}\right)}{\sqrt{-F}}$.

Recalling $\gamma=-\lambda_{3}^{2}$, we deduce $2=c_{3}^{2}=2 \varepsilon \sinh ^{2}\left(\frac{y_{1}}{2}\right)$ so that $\cosh y_{1}=\frac{2+\varepsilon}{\varepsilon}$, whence by definition of $y_{1}, F=-(1+\varepsilon)$ in accordance with (58).

We now proceed to a discussion of case (b). From (60) and (62), we get $T \equiv 0$ and $S \equiv 0$, respectively. This implies $\gamma=0$ so that $\lambda_{1} \neq 0$ by the above. We therefore obtain

$$
W(x)=\frac{\sqrt{-F}}{\sqrt{-2 \lambda_{1}} \cosh y(x)}
$$

so that the boundary condition $W( \pm 1)=\varepsilon$ leads to $\lambda_{1}=\frac{F}{2 \varepsilon^{2} \cosh ^{2} y_{1}}$. We calculate

$$
c_{1}^{2}=\int_{-1}^{1} \frac{W^{2}}{1+\varepsilon-x^{2}} \mathrm{~d} x=-\frac{\sqrt{-F}}{2 \lambda_{1}} \tanh y_{1}
$$

Recall that in this particular case also $\lambda_{1}=-\frac{2}{c_{1}^{4}}$ by (53) so that $y_{1}=\operatorname{arsinh} \frac{2}{\varepsilon}$ and we arrive at estimate (58) using

$$
\operatorname{arsinh} x=\ln \left(x+\sqrt{x^{2}+1}\right) \text { and } \operatorname{artanh} x=\frac{1}{2} \ln \frac{1+x}{1-x} .
$$

Let us continue with case (c). From (60) we get $T \equiv 0$, whereas $M_{1}=0$ implies $M_{2}=0$ and thus $\lambda_{1}=0$ by (53). On the other hand, we get $f^{\prime}(0)=0$ from (61) so that by (63) we have $\gamma=0$, a contradiction, because we have seen in the previous subsection that $\lambda_{1}$ and $\gamma$ cannot vanish simultaneously.

Finally, we discuss case (d). As before, we get $T \equiv 0$ and thus by (43) $\lambda_{2}=0$ as $c_{3} \neq 0$ ensures $S \neq \equiv 0$. Equation (62) then leads to $\lambda_{3}=-\frac{\sqrt{2} c_{3}^{3}}{c_{1}^{2}}$. From this, we obtain $\int_{-1}^{1} \mathrm{e}^{2 U} \mathrm{~d} x=\frac{c_{1}^{2}}{c_{3}^{2}}$, where we used (48) and $c_{3} \neq 0$. Also, $\gamma=-\lambda_{3}^{2}$ so that $\gamma=-\frac{2 c_{3}^{6}}{c_{1}^{4}}$. In particular, $I_{\varepsilon}[S, T=0, U]=I_{\varepsilon}[0,0, U]+\frac{c_{3}^{4}}{2 c_{1}^{2}}>I_{\varepsilon}[0,0, U]$. This contradicts $[S, T=0, U]$ being a global minimizer of $I_{\varepsilon}$.

5.7. Estimating the minimal value of $I_{\varepsilon}$ : the case $c_{2} \neq 0$. Finally let $c_{2} \neq 0$. If $\lambda_{1}=0$ were possible, then by (53) $M_{2}=0$ so that $g^{\prime}(0)=0$ and thus $T \equiv 0$ follow from (59), (60). Equation (61) then tells us that $f^{\prime}(0)=0$ and whence $S \equiv 0$, so that also $\gamma=0$, in contradiction to the above exclusion of $\lambda_{1}=\gamma=0$. Thus, $\lambda_{1} \neq 0$ which implies 
both $M_{2} \neq 0$ and $M_{1} \neq 0$. Using again (60), (62), and (53), we obtain:

$$
\begin{aligned}
& \lambda_{1}=-\frac{\left(\sqrt{4-c_{3}^{4}}-2\left|c_{2}\right|\right)^{2}}{2 c_{1}^{4}}, \\
& \lambda_{2}=-\frac{2\left(\sqrt{4-c_{3}^{4}}-2\left|c_{2}\right|\right)}{c_{1}^{2}} \operatorname{sign}\left(c_{2}\right), \\
& \lambda_{3}=-\frac{\sqrt{2}\left(\sqrt{4-c_{3}^{4}}-2\left|c_{2}\right|\right) c_{3}^{3}}{\sqrt{4-c_{3}^{4}} c_{1}^{2}} .
\end{aligned}
$$

Rewrite $S, T$ in terms of $A(x):=\lambda_{2} \int_{0}^{x} \mathrm{e}^{2 U(t)} \mathrm{d} t, A_{1}:=A(1)$ and use $W( \pm 1)=\varepsilon$, equation (52), and our definition of $y_{1}$ as well as symmetry of $U$ to obtain

$$
\begin{aligned}
& T(x)=\frac{\lambda_{3}}{\lambda_{2}}\left[\frac{\cos A(x)}{\cos A_{1}}-1\right], \\
& S(x)=-\lambda_{3} \frac{\cos A(x)}{\cos A_{1}} \mathrm{e}^{2 U(x)} \text {, } \\
& \gamma=-\frac{\lambda_{3}^{2}}{\cos ^{2} A_{1}} \\
& c_{1}^{2}=-\frac{\sqrt{-F}}{2 \lambda_{1}}\left[\frac{\sqrt{\gamma^{2}+8 \lambda_{1} F} \sinh y_{1}}{\sqrt{\gamma^{2}+8 \lambda_{1} F} \cosh y_{1}-\gamma}\right. \\
& \left.+\frac{2 \gamma}{\sqrt{8 \lambda_{1} F}} \arctan \left(\frac{\gamma+\sqrt{\gamma^{2}+8 \lambda_{1} F}}{\sqrt{8 \lambda_{1} F}} \tanh \frac{y_{1}}{2}\right)\right] \text {, } \\
& c_{2}=-\frac{\lambda_{3}^{2}}{\lambda_{2}^{2}} \frac{A_{1}-\sin A_{1} \cos A_{1}}{\cos ^{2} A_{1}} \text {, } \\
& c_{3}=-\sqrt{2} \frac{\lambda_{3}}{\lambda_{2}} \tan A_{1} \text {, } \\
& A_{1}=\frac{\lambda_{2}}{\sqrt{-2 \lambda_{1}}} \arctan \left(\frac{\gamma+\sqrt{\gamma^{2}+8 \lambda_{1} F}}{\sqrt{8 \lambda_{1} F}} \tanh \frac{y_{1}}{2}\right) \text {, } \\
& \varepsilon=\frac{2 F}{\gamma-\sqrt{\gamma^{2}+8 \lambda_{1} F} \cosh y_{1}}, \\
& y_{1}=\frac{2 \sqrt{-F}}{\sqrt{1+\varepsilon}} \operatorname{artanh} \frac{1}{\sqrt{1+\varepsilon}} \text {. }
\end{aligned}
$$

Now set $\phi:=\arccos \frac{-\gamma}{\sqrt{\gamma^{2}+8 \lambda_{1} F}} \in\left(0, \frac{\pi}{2}\right)$ which is well-defined as $\lambda_{1} \cdot F>0$. Using this new constant, Eqs. (67) through (75) take on a simpler form. In particular, these equations lead to $0<\left|A_{1}\right| \leq \phi<\frac{\pi}{2}$ and

$$
c_{1}^{2}=\frac{4 \tan \phi \cos A_{1}\left|\sin A_{1}-A_{1} \cos A_{1}\right|}{\sqrt{-F} \sin ^{4} A_{1}}, \quad c_{3}= \pm \sqrt{2 \cos A_{1}} .
$$


For the Lagrange multipliers, we get

$$
\begin{aligned}
& \lambda_{1}=\frac{F \sin ^{4} A_{1}}{8 \tan ^{2} \phi \cos ^{2} A_{1}}, \\
& \lambda_{2}=\frac{4\left(\sin A_{1}-A_{1} \cos A_{1}\right)}{c_{1}^{2} \sin ^{2} A_{1}}, \\
& \lambda_{3}=\mp \frac{4}{c_{1}^{2}} \cos ^{\frac{3}{2}} A_{1} \frac{\left|\sin A_{1}-A_{1} \cos A_{1}\right|}{\sin ^{3} A_{1}} .
\end{aligned}
$$

With the above expressions, we obtain

$$
\varepsilon=\frac{2 \cos A_{1} \sin ^{2} \phi}{\sin ^{2} A_{1} \cos \phi\left(\cosh y_{1}+\cos \phi\right)}
$$

As $\frac{\sin ^{2} x}{\cos x}$ is monotonically increasing on $\left(0, \frac{\pi}{2}\right)$ and $\left|A_{1}\right| \leq \phi$, we have $\varepsilon \geq \frac{2}{\cosh y_{1}+1}$ or in other words

$$
y_{1} \geq \operatorname{arcosh}\left(\frac{2}{\varepsilon}-1\right) .
$$

This implies

$$
\sqrt{-F} \geq \frac{\sqrt{1+\varepsilon} \operatorname{arcosh}\left(\frac{2}{\varepsilon}-1\right)}{2 \operatorname{artanh} \frac{1}{\sqrt{1+\varepsilon}}}
$$

where we have used (75). Recall $\operatorname{arcosh} x=\ln \left(x+\sqrt{x^{2}-1}\right)$ to deduce (58) also in the discussed case $c_{2} \neq 0$.

5.8. The limit $\varepsilon \rightarrow 0$. We conclude as promised that for $c_{2}=0$ cases (c) and (d) cannot apply for the minimizer $(S, T, U)$, whereas in the remaining cases (a) and (b), as well as for $c_{2} \neq 0$, we can estimate using (57) and (58) that

$$
\begin{aligned}
I_{\varepsilon}[S, T, U] & \geq 1+\sqrt{1+\varepsilon}\left[\left(1-\frac{2 \ln \frac{2+\varepsilon}{2-\varepsilon}}{\ln \frac{(2+\varepsilon)^{2}}{\varepsilon}}\right)^{2}-1\right] \operatorname{artanh} \frac{1}{\sqrt{1+\varepsilon}} \\
& \geq 1-2 \sqrt{1+\varepsilon} \ln \frac{2+\varepsilon}{2-\varepsilon} .
\end{aligned}
$$

We now study the limit $\varepsilon \rightarrow 0$. For any $(S, T, U) \in X, c_{2}[S, T, U], c_{3}[S, T, U]$ and thus $M_{1}[S, T, U]$ are independent of $\varepsilon, \lim _{\varepsilon \rightarrow 0} c_{1}^{\varepsilon}[S, T, U]=c_{1}^{0}[S, T, U]$ and thus $\lim _{\varepsilon \rightarrow 0} M_{2}^{\varepsilon}[S, T, U]=M_{2}^{0}[S, T, U]$ so that

$\left|I[S, T, U]-I_{\varepsilon}[S, T, U]\right| \leq \frac{\varepsilon}{2} \int_{-1}^{1} U^{\prime}(x)^{2} \mathrm{~d} x+\left|\frac{M_{2}[S, T, U]^{2}}{2}-\frac{M_{2}^{\varepsilon}[S, T, U]^{2}}{2}\right| \rightarrow 0$ 
as $\varepsilon \rightarrow 0$, i.e. $I_{\varepsilon}[S, T, U]$ is continuous at $\varepsilon=0$ for fixed $(S, T, U)$. This finally leads us to an esimate of the original functional $I$. We obtain

$$
\begin{aligned}
I[S, T, U] & =\lim _{\varepsilon \rightarrow 0} I_{\varepsilon}[S, T, U] \\
& \geq \liminf _{\varepsilon \rightarrow 0} I_{\varepsilon}\left[S^{*}, T^{*}, U^{*}\right] \\
& \geq \liminf _{\varepsilon \rightarrow 0}\left(1-2 \sqrt{1+\varepsilon} \ln \frac{2+\varepsilon}{2-\varepsilon}\right) \\
& =1,
\end{aligned}
$$

where $\left(S^{*}, T^{*}, U^{*}\right)$ denotes the global minimizer of $I_{\varepsilon}$.

This proves the claim of Lemma 2 and therefore the inequality (2).

Finally, after we have seen that the functional $I$ has a lower bound of 1 , we can ask the question of whether there exist functions $S, T$, and $U$ for which $I$ takes on this value. The investigation of this question together with a discussion of the meaning of $I$ in the context of degenerate black holes can be found in Appendix A.

\section{Discussion}

With techniques from the calculus of variations, we have shown that the inequality $p_{J}^{2}+p_{Q}^{2}<1$ holds for axisymmetric and stationary sub-extremal black holes with surrounding matter in full Einstein-Maxwell theory.

In particular, we have proved the inequality $I[S, T, U] \geq 1$ for the functional $I$ defined in (34). As $I$ could not directly be seen to have a local minimizer, we introduced a family of approximating functionals $I_{\varepsilon}$ which could be shown to have one.

Together with a theorem for degenerate black holes in [1], we can deduce the following.

Theorem 1. Consider Einstein-Maxwell spacetimes with vanishing cosmological constant. Then, for every axisymmetric and stationary sub-extremal black hole with arbitrary surrounding matter we have the inequality

$$
(8 \pi J)^{2}+\left(4 \pi Q^{2}\right)^{2}<A^{2} .
$$

If the axisymmetric and stationary black hole is degenerate, the equation

$$
(8 \pi J)^{2}+\left(4 \pi Q^{2}\right)^{2}=A^{2}
$$

holds.

Observe that the assumptions for the result in [1] which has been used here have been weakened, see Appendix A.

Theorem 1 provides a remarkable relation between the geometrical concept of the existence of trapped surfaces and the physical black hole properties described by rotation rate $p_{J}$ and charge rate $p_{Q}$. We see that "physically reasonable" (sub-extremal) black holes cannot rotate "too fast" and cannot be charged "too strongly".

Finally, our results shed new light on the notions of sub-extremality and extremality of axisymmetric and stationary black holes. Any sub-extremal black hole in the sense of [3] (the notion of which we have used throughout this paper) is also sub-extremal in the sense that $p_{J}^{2}+p_{Q}^{2}<1$. In fact, $p_{J}^{2}+p_{Q}^{2}=1$ holds in the degenerate limit, for which reason we may call these black holes "extremal". 
Acknowledgement. We would like to thank Herbert Pfister for many valuable discussions and John Head for commenting on the manuscript. This work was supported by the Deutsche Forschungsgemeinschaft (DFG) through the Collaborative Research Centre SFB/TR7 "Gravitational wave astronomy" and by the International Max Planck Research School for "Geometric Analysis, Gravitation and String Theory".

Open Access This article is distributed under the terms of the Creative Commons Attribution Noncommercial License which permits any noncommercial use, distribution, and reproduction in any medium, provided the original author(s) and source are credited.

\section{A. Remarks on Degenerate Black Holes}

In order to discuss extremal black holes (as done in [1]) and to get an idea of the meaning of the appearing functional $I$, one can apply similar techniques as used in Sec. 5 to $I$ itself to derive Euler-Lagrange equations and a complete characterization of the minimizers of $I$. As minimizers of $I$ need not be limits of minimizers of $I_{\varepsilon}$, this renewed analysis is necessary.

It turns out that the Euler-Lagrange equations for $S$ and $T$ are just as before, cf. (43) and (44). Moreover, there again exists an integrating factor for the Euler-Lagrange equation for $U$ leading to

$$
-1=-\left(1-x^{2}\right)^{2} U^{\prime 2}+2 x\left(1-x^{2}\right) U^{\prime}+2 \lambda_{1} \mathrm{e}^{4 U}\left(1-x^{2}\right)^{2}-x^{2}+\gamma\left(1-x^{2}\right) \mathrm{e}^{2 U},
$$

where $\gamma$ is defined as in (52). Introducing $W(x):=\left(1-x^{2}\right) \mathrm{e}^{2 U(x)}$ on the interior $(-1,1)$, we find that Eq. (55) holds on $(-1,1)$ with $\varepsilon=0$ and $F=-1$. Discussing the radicand in (55) as before, we see that it vanishes at at most one inner point. Assuming non-vanishing of the radicand and integrating the equation on $(-1+\delta, 1-\delta)$ for some $\delta>0$ leads to a contradiction as the unique solution $U$ derived from $W$ diverges as $\delta \rightarrow 0$ while we know that the smooth solution $U$ exists on the whole interval by the same bootstrap argument as sketched above. Thus we know that there exists exactly one interior zero of the radicand and we can integrate the equation as before to obtain

$$
\mathrm{e}^{2 U(x)}=\frac{2}{(1+\gamma) x^{2}+1-\gamma},
$$

and the consistency condition

$$
\gamma^{2}-8 \lambda_{1}=1,
$$

using the boundary values for $U, U( \pm 1)=0$. In other words, $U$ belongs to a family parametrized by $\gamma \in[-1,0]$.

Proceeding as above, we find that $S$ and $T$ are given by

$$
\begin{aligned}
& S(x)= \pm\left[(-\gamma)^{\frac{3}{2}}+\sqrt{1-\gamma^{2}}|T(x)|\right] \mathrm{e}^{2 U}, \\
& T(x)= \pm \sqrt{-\gamma\left(1-\gamma^{2}\right)} \frac{1-x^{2}}{1-\gamma+(1+\gamma) x^{2}}
\end{aligned}
$$

with $\gamma \in[-1,0]$. The signs of $S$ and $T$ can be chosen independently of each other. It can a posteriori be seen that all functions $S, T, U$ of this form with $\gamma \in[-1,0]$ in fact satisfy

$$
I[S, T, U]=1
$$


so that we have identified all minimizers of $I$. Moreover, one can show that the Lagrange parameters $\lambda_{1}, \lambda_{2}, \lambda_{3}$ can explicitly be expressed as

$$
\lambda_{1}=-\frac{1}{8}\left(1-\gamma^{2}\right), \quad \lambda_{2}= \pm \sqrt{1-\gamma^{2}}, \quad \lambda_{3}= \pm(-\gamma)^{\frac{3}{2}},
$$

where again the signs are not correlated.

By comparison with [1], one finds that these are exactly the functions $S, T$, and $U$ arising in the context of arbitrary degenerate black holes with surrounding matter. ${ }^{8}$ Moreover, the differential equations characterizing $S, T$, and $U$ in [1] are exactly the Euler-Lagrange equations of $I$ derived in this paper where the Lagrange parameters in (78) correspond to the constants appearing in [1].

We arrive at two conclusions: First, our analysis dispenses with additional assumptions made in [1], namely equatorial symmetry and the existence of a continuous sequence of spacetimes, leading from the Kerr-Newman solution in electrovacuum to the discussed black hole solution. The latter was necessary to assure uniqueness (up to a parameter) of the solution to the horizon equations in [1].

As a matter of fact, any smooth solution of the equations in [1] is a minimizer of $I$ as can be seen by solving the equations as done above and using the relations in (78) between $\gamma$ and the Lagrange parameters. Thus, any solution of these equations is automatically equatorially symmetric and of the form assumed in [1]. Hence, the unnecessary assumptions of [1] can be dropped.

Secondly, we see that the functional $I$ plays the role of a "primitive" of the Einstein equations on the event horizon of degenerate black holes: Remarkably, the Euler-Lagrange equations corresponding to $I$ lead uniquely to the electromagnetic and metric potentials $S, T$, and $U$ belonging to degenerate black holes.

\section{References}

1. Ansorg, M., Pfister, H.: A universal constraint between charge and rotation rate for degenerate black holes surrounded by matter. Class. Quantum Grav. 25, 035009 (2008)

2. Bardeen, J.M.: Rapidly rotating stars, disks, and black holes. In: Black holes (Les Houches), deWitt, C., deWitt, B.S., ed., London: Gordon and Breach, 1973, pp. 241-289

3. Booth, I., Fairhurst, S.: Extremality conditions for isolated and dynamical horizons. Phys. Rev. D 77, 084005 (2008)

4. Buttazzo, G., Giaquinta, M., Hildebrandt, S.: One-dimensional variational problems. Oxford: Clarendon Press, 1998

5. Carter, B.: Black hole equilibrium states. In: Black Holes (Les Houches), deWitt, C., deWitt, B.S., ed., London: Gordon and Breach, 1973, pp. 57-214

6. Evans, L.C.: Partial Differential Equations. Providence, RI: Amer. Math. Soc. 2002

7. Hennig, J., Ansorg, M., Cederbaum, C.: A universal inequality between the angular momentum and horizon area for axisymmetric and stationary black holes with surrounding matter. Class. Quantum Grav. 25, 162002 (2008)

8. Rauch, J.: Partial Differential Equations. Berlin: Springer, 1991

9. Yosida, K.: Functional Analysis. Berlin: Springer, 1995

Communicated by P.T. Chruściel

8 The parameter $\alpha \in[-1,1]$ in [1] is related to $\gamma$ via $\gamma=-\left(1-\alpha^{2}\right) /\left(1+\alpha^{2}\right)$. 